\title{
Alterations in mucosa-associated microbiota in the stomach of patients with gastric cancer
}

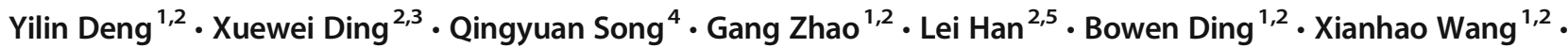 \\ Xishan $\mathrm{HaO}^{1,2} \cdot \mathrm{Hui}^{\mathrm{Li}}{ }^{1,2}$
}

Accepted: 15 February 2021 / Published online: 26 March 2021

(C) The Author(s) 2021

\begin{abstract}
Purpose The purpose of this study was to characterize alterations in mucosa-associated microbiota in different anatomical locations of the stomach during gastric cancer progression and to identify associations between Helicobacter pylori infection and gastric microbial changes in patients with gastric cancer.

Methods Twenty-five $H$. pylori negative subjects with chronic gastritis and thirty-four subjects with gastric cancer were recruited, including $H$. pylori negative and positive patients with tumors in the antrum and the corpus. Gastric mucosa-associated microbiota were determined by $16 \mathrm{~S}$ ribosomal RNA gene sequencing using a 454 sequencing platform.

Results We found that individuals with chronic gastritis from three different anatomical sites exhibited different microbiota compositions, although the microbial alpha diversity, richness and beta diversity were similar. Compared to patients with chronic gastritis, the gastric microbiota compositions were significantly different at the order level in the antrum and the corpus of patients with gastric cancer, which was dependent on the H. pylori infection status. Microbial alpha diversity and species richness, however, were similar between chronic gastritis and gastric cancer cases and independent of $H$. pylori status. The microbial community structure in patients with gastric cancer was distinct from that in patients with chronic gastritis. In addition, we found that the presence of $H$. pylori markedly altered the structure in gastric corpus cancer, but only mildly affected the antrum.

Conclusion Our data revealed distinct niche-specific microbiota alterations during the progression from gastritis to gastric cancer. These alterations may reflect adaptions of the microbiota to the diverse specific environmental habitats in the stomach, and may play an important, as yet undetermined, role in gastric carcinogenesis.
\end{abstract}

Keywords Chronic gastritis gastric cancer microbiota Helicobacter pylori

\section{Introduction}

The human gastrointestinal tract harbors a broad range of microbiota which contribute to the establishment of a symbiotic

Hui Li

lihui@tjmuch.com

1 Department of Gastrointestinal Cancer Biology, Tianjin Medical University Cancer Institute \& Hospital, Tianjin 300060, China

2 National Clinical Research Center for Cancer, Tianjin 300060, China

3 Department of Gastric Cancer, Tianjin Medical University Cancer Institute and Hospital, Tianjin 300060, China

4 Department of Computer and Information Science, University of Pennsylvania, PA 19104 Philadelphia, USA

5 Cancer Molecular Diagnostics Core, Tianjin Medical University Cancer Institute \& Hospital, Tianjin 300060, China relationship with the host [1]. This relationship provides a nutrient-rich environment for the microbiota, while playing important roles in maintaining physiological balances and health of the host [2-4]. The microbiota in the stomach form a complex community which can be shaped by various factors. Due to the harsh environment in the stomach, it was commonly assumed to be sterile until the discovery and isolation of Helicobacter pylori [5]. Recently, the profiles of gastric microbiota have gradually been unveiled by virtue of next generation sequencing (NGS) technologies. Proteobacteria, Firmicutes, Bacteroidetes, Actinobacteria and Fusobacteria are the most commonly detected phyla in the stomach [6]. Alterations in richness or diversity of microbiota may result in dysbiosis. Increasing evidence indicates that dysbiosis of the gastrointestinal microbiota is associated with gastric cancer [7] as well as with a wide variety of other disorders, including inflammatory bowel disease [8], liver pathology [9], autoimmune disease [10] and brain dysfunction [11]. 
Gastric cancer is one of the leading causes of cancer-related death worldwide, especially in eastern Asia [12]. A complex interaction between the host genetic background and environmental factors contributes to the development of gastric cancer [13]. Gastric adenocarcinoma comprises more than $90 \%$ of all malignant tumors in the stomach, including cardia and non-cardia gastric cancer according to the site where the tumor arises. Risk factors associated with non-cardia gastric cancer are $H$. pylori infection, low socio-economic conditions, and dietary habits such as low intake of vegetables and fruits and high consumption of salty and smoked food. Obesity and gastroesophageal reflux disease are exclusive factors for cardia gastric cancer [14].

H. pylori has been designated as a class I carcinogen and has been identified as the main risk factor for gastric carcinoma. Persistent chronic inflammation induced by $H$. pylori infection is a decisive factor in the progression of gastric cancer [13]. However, only a small portion $(<3 \%)$ of $H$. pylori infected patients eventually develop gastric cancer [13]. It has been reported that complex gastric microbiota may significantly accelerate the onset of precancerous gastric lesions rather than H. pylori infection alone in a transgenic model of gastric carcinogenesis, i.e., insulin-gastrin (INS-GAS) mice [15]. Antimicrobial therapy significantly delayed the onset of gastrointestinal intraepithelial neoplasia (GIN) in both $H$. pylori-infected and $H$. pylori-free INS-GAS mice [15]. In addition, it was found that gastric colonization with a restricted microbiota promoted neoplastic lesions in H. pylori-mono-associated INS-GAS mice [16]. So, several lines of evidence indicate a relationship between gastric dysbiosis and H. pylori infection, and the development of gastric cancer.

However, due to the different techniques used, the complexity of bioinformatic analyses and the intricacy of gastric cancer, there is currently no consensus on the drivers or modifiers in the microbiome that mediate the development of gastric cancer. It is well known that long-term inflammation in the stomach may lead to chronic gastritis, atrophy, intestinal metaplasia, dysplasia and, ultimately, cancer [17]. The purpose of this study was to characterize alterations in mucosa-associated microbiota during gastric cancer development and to delineate the influence of $H$. pylori infection on the gastric microbial community in gastric cancer patients. Information on the microbial profile in the stomach was obtained by analyzing microbiota in different anatomic sites (antrum, corpus and cardia). Our findings underscore dysbiosis as a risk factor or consequence of gastric cancer and a concomitant influence of $H$. pylori infection on gastric cancer development.

\section{Materials and methods}

\subsection{Patients and sample collection}

This study was approved by the Clinical Research Ethics Committees of Tianjin Medical University Cancer Institute and Hospital, Tianjin, China. Written informed consent was obtained from all individuals included in this study. All subjects enrolled in this cohort resided in Tianjin, China. Patients were recruited at Tianjin Medical University Cancer Institute and Hospital. H. pylori infection status was examined by urease test. Twenty-five individuals (12 female and 13 male, age range: 45 to 70 ) with chronic superficial gastritis were recruited in this study. All individuals in this group were H. pylori negative. Gastric mucosal biopsy samples were obtained from lesions including the antrum $(n=10)$, corpus $(n=7)$ and cardia $(n=8)$ during standard upper gastroenterology endoscopic examination. In addition, thirty-four patients (10 female and 24 male, age range: 46 to 75 ) with gastric cancer were enrolled in this study. All of them were diagnosed as gastric adenocarcinoma, including patients with cancer in the antrum $(n=19)$ and corpus $(n=15)$. Cancer stages included stage I $(n=3)$, stage II $(n=7)$, stage III $(n=23)$ and stage IV $(n=1)$. Gastric cancer biopsy tissues were obtained from the sites of cancer lesion during surgery. Five patients with gastric antrum cancer and four patients with gastric corpus cancer were H. pylori positive. Patients who received antibiotics, acid blockers (including proton pump inhibitors and histamine-2 receptor antagonists), anti-inflammatory drugs or probiotic treatment during the past 6 months were excluded. In addition, none of the patients received chemotherapy or radiotherapy prior to the surgery.

\subsection{S rRNA gene sequencing and microbiota profile analysis}

Frozen gastric mucosa biopsy samples and gastric cancer tissues were sent to the Research and Testing Laboratory, LLC (Lubbock, TX, USA) for metagenomic analyses. Total genomic DNA was extracted and sequenced using primers targeting the $16 \mathrm{~S}$ ribosomal regions on a Roche 454 sequencing platform. Sequence data were processed using a Research and Testing pipeline that is described at http://www. researchandtesting.com/docs/Data_Analysis_Methodology. pdf. Raw DNA sequencing data were analyzed using QIIME (http://www.qiime.org). The data analysis pipeline consisted of two major stages, a denoising and chimera detection stage and a microbial diversity analysis stage. The process of denoising was used to obtain clean reads by discarding adaptors, short and low-quality sequences. Chimera detection and removal by executing UCHIME on the filtered data served as a denoising method. Next, sequences were clustered into OTUs (Operational Taxonomic Units) using the UPARSE algorithm with at least $97 \%$ similarity. Taxonomic information was assigned to centroid sequences from each cluster by the RDP (ribosome database project) classifier against the NCBI database, after which diversity parameters were examined from two perspectives. Alpha diversity analysis was used to describe the richness and diversity of the 
microbiota within the sample. First, overall richness was quantified using the Chao 1 richness estimator. Second, overall diversity was expressed as Shannon Diversity, which was determined by both richness and evenness, the distribution of abundance among distinct taxa. Beta diversity referred to the diversity among samples, which was measured by covariance matrix and visualized via principal component analysis (PCA). Specific microbial taxa were identified through a linear discriminant analysis effect size (LEfSe) algorithm, performed on the Galaxy web-based interface (http:// huttenhower.sph.harvard.edu/galaxy). Taxa with a linear discriminant analysis (LDA) $>3.0$ and an alpha value $<0.05$ were considered significant.

\subsection{Statistical analysis}

Data that were not normally distributed are presented as median values and ranges ( $\min$ to max). Mann-Whitney $U$ test was used to determine the difference between two sample groups. Multiple sample groups were analyzed using Kruskal-Wallis test. Data that were normally distributed are presented as mean \pm SEM, and one-way ANOVA was used to analyze differences. All tests were performed using GraphPad Prism 8.0.1. $P<0.05$ was considered statistically significant. Heatmaps and PCAs were generated using Python.

\section{Results}

\subsection{Gastric mucosa-associated microbiomes differ in different anatomical locations of individuals with chronic gastritis}

Although microbial profiles in the stomach have been reported before, knowledge on whether different anatomical sites harbor different microbiota, especially under inflammatory conditions, is limited. Therefore, we first analyzed gastric mucosa-associated microbiota in different anatomical sites including the antrum, corpus and cardia in patients with chronic gastritis. The most representative phyla were Proteobacteria, Firmicutes, Bacteroidetes, Actinobacteria and Fusobacteria, representing over $95 \%$ of the total phylum (Table 1). The relative abundances of Proteobacteria and Firmicutes were significantly different among the three different anatomical sites $(p<0.05$, Table 1). Proteobacteria were predominant in the corpus, whereas those of Proteobacteria and Firmicutes were at comparable levels in the antrum and cardia (Table 1). At the genus level, Citrobacter $(31.30 \pm 2.65 \%)$ was the most abundant taxa in the antrum, followed by Streptococcus $(28.64 \pm 1.22 \%)$ and Rothia $(16.95 \pm 1.82 \%)$. However, in the corpus, the major genus was Acinetobacter $(78.06 \pm 3.30 \%)$, with a relative abundance $>90 \%$ in over half of all samples. Conversely, the overrepresented genus in the
Table 1 Relative abundances of major bacterial phyla in chronic gastritis subjects

\begin{tabular}{lllll}
\hline Taxonomy & Antrum (\%) & Corpus (\%) & Cardia (\%) & $P$ value \\
\hline Proteobacteria & 38.03 & 80.18 & 48.99 & $\mathbf{0 . 0 4}$ \\
Firmicutes & 36.94 & 1.69 & 28.71 & $\mathbf{0 . 0 1}$ \\
Actinobacteria & 14.63 & 1.36 & 5.6 & 0.11 \\
Bacteroidetes & 2.95 & 7.26 & 2.13 & 0.58 \\
Fusobacteria & 1.7 & 0.38 & 1.26 & 0.91 \\
\hline
\end{tabular}

The $P$ values are calculated using Kruskal-Wallis test and marked in bold if $<0.05$

cardia was Klebsiella $(30.99 \pm 3.36 \%)$, followed by Streptococcus $(18.68 \pm 2.15 \%)$ and Escherichia $(16.19 \pm$ 2.18\%) (Fig. 1). Consistent with the findings at the genus level, Acinetobacter $s p$ and Klebsiella $s p$ were the only significantly abundant species enriched in the corpus and the cardia, respectively (Supplementary Fig. 1). Weissella confuse, a member of the genus Weissella, was detected as a high-abundance species in antrum-predominant gastritis (Supplementary Fig. 1). However, a large degree of intersubject variability was observed, with relative abundances ranging from 0 to $98.89 \%$.

The relative abundance of $H$. pylori in all subjects included in this group was extremely low. According to a previous report, $H$. pylori sequencing positive was referred to as those samples with a relative abundance $>1 \%$ [18]. In this group, the H. pylori sequence status of all samples was negative $(<1 \%)$, consistent with the results of the conventional urease test.

To evaluate differences in microbiota structure of chronic gastritis from three different anatomical sites, we measured microbial alpha and beta diversities. Chao 1 and Shannon indexes were used to measure species richness and alpha diversity, respectively. We found that there was no significant difference in microbial richness and alpha diversity within the three different anatomical sites (Fig. 2a). Differences in microbial community structures of chronic gastritis from three different anatomical sites were assessed by beta diversity and visualized by PCA plot. A PCA plot based on covariance matrix revealed that most samples from the three different anatomical locations overlapped, although the cardia cases were more dispersed than the those of other two sites (Fig. 2b). The three different anatomical sites were not clustered separately, suggesting similarity in the overall community structure of chronic gastritis in the three different anatomical locations.

To further assess specific taxa associated with each anatomical site, we conducted LEfSe analysis. Overall, there were 20 taxa that were differentially abundant in gastritis from the three different anatomical sites. Nine of them were enriched in antrum gastritis, including the phylum Firmicutes with its specific genus Streptococcus, and the phylum Proteobacteria with its specific genera Stenotrophomonas and Citrobacter. The genus 
Fig. 1 Microbial composition analysis of chronic gastritis patients. a Heatmap of gastric mucosa-associated microbiota with relative abundance over $1 \%$ in all subjects. b Bar plots of relative abundances of gastric mucosa-associated microbiota. c Representative microbiota among chronic gastritis subjects from three different anatomical locations

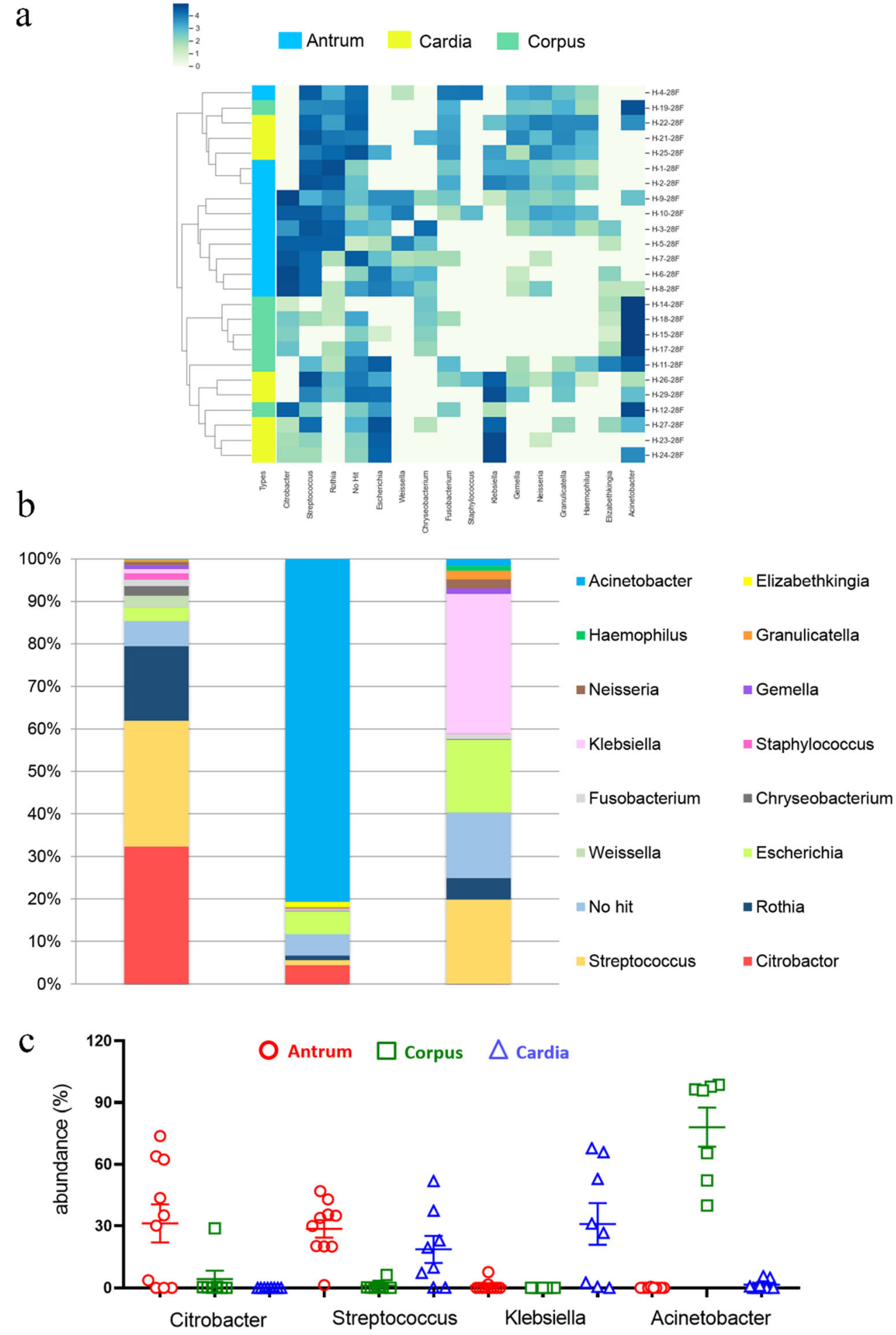

Klebsiella, within the phylum Proteobacteria, was the only enriched taxa in the cardia. All taxa enriched in the corpus belonged to the phylum Proteobacteria, including the genera Pseudomonas, Acinetobacter and Methylobacterium (Fig. 3). These results indicate that the most relevant taxa are responsible for the differences in chronic gastritis from the three different anatomical sites, and that all of them belong to the phyla Firmicutes and Proteobacteria.

Taken together, these data suggest that the composition of the microbiota in the different anatomical sites varies, but that there are no differences in microbial diversity and evenness in individuals with chronic gastritis. 

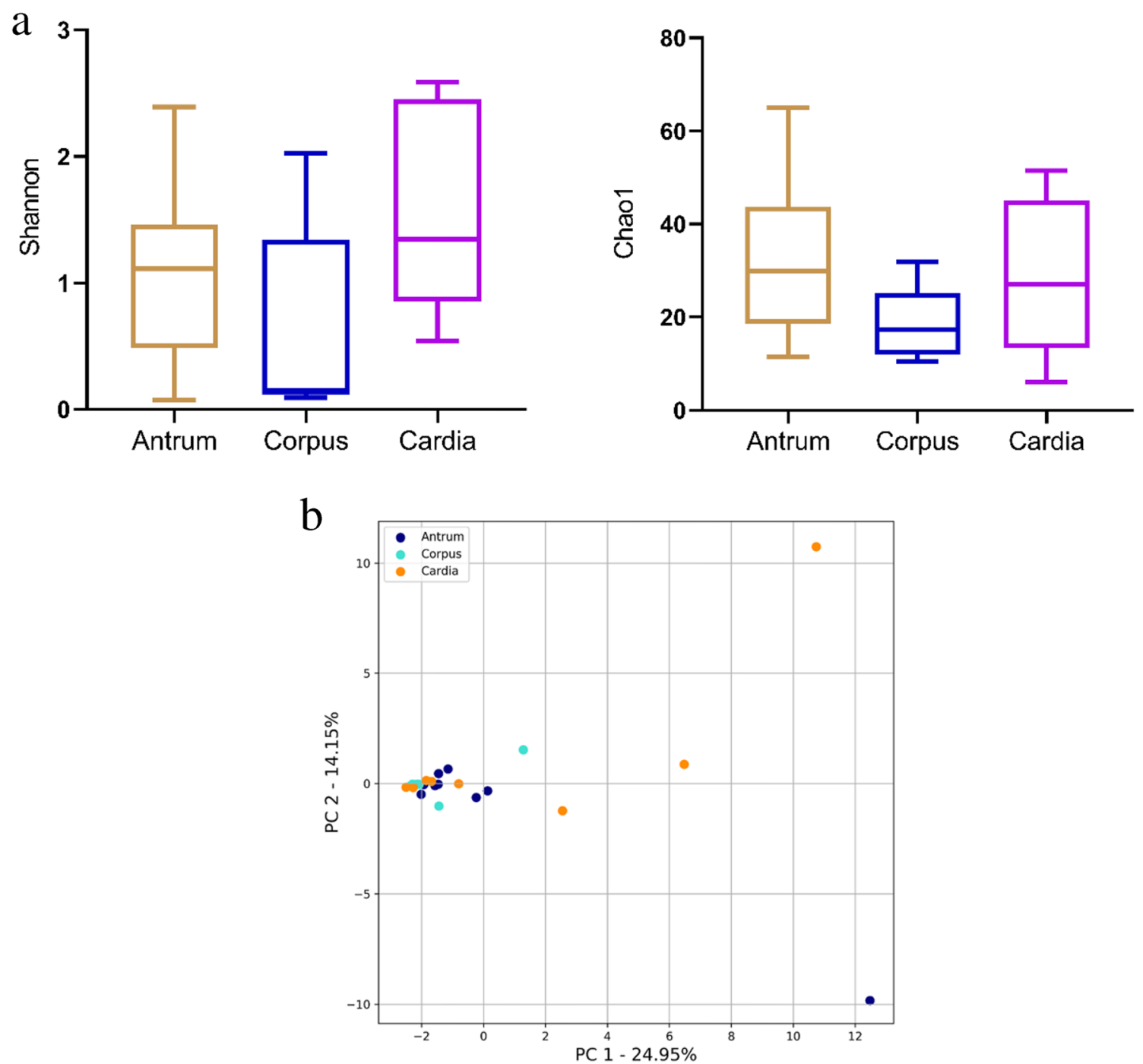

Fig. 2 Microbial community structure analysis of chronic gastritis patients from three different anatomical positions. a Microbial alpha diversity and richness analysis $(p>0.05)$. b Principal component analysis (PCA)

\subsection{Differences in the gastric mucosa-associated microbiome in $H$. pylori negative patients with gastric cancer}

Since we identified differences in the microbial composition in different anatomical sites in individuals with gastritis, we next examined how the microbiota profile in different anatomical sites may change from gastritis to gastric cancer. Overall, we found that there were only minor differences in major bacteria phyla between $H$. pylori negative gastritis and gastric cancer individuals from the corpus and antrum. Although the ranking of five main phyla differed slightly between these two anatomical sites, Proteobacteria was the dominant phylum in gastric cancer, with a slight increase compared to gastritis (Tables 2 and 3). At the order level, Pseudomonadales $(96.87 \pm 0.38 \%$ vs $77.34 \pm$ $2.74 \%, p=0.02$ ) became the most abundant flora in gastric corpus cancer with a relative abundance $>90 \%$ in all subjects of this group (Fig. 5). The increase was more pronounced in gastric antrum cancer, Pseudomonadales was up to $50.48 \pm 2.65 \%$ (vs $0.26 \pm 0.36 \%, p<0.01$ ) with a relative abundance $>50 \%$ in over half of all subjects (Fig.4). However, Enterobacteriales was less abundant in both gastric antrum $(15.20 \pm 1.70$ vs $36.99 \pm 3.65 \%$, $p=0.01)$ (Fig. 4$)$ and corpus (5.05 $\pm 1.83 \%$ vs $13.39 \pm 1.79 \%$, $p<0.01$ ) cancer (Fig. 5). The proportion of Actinobacteria was found to be decreased in gastric antrum cancer in comparison to antrum predominant gastritis, while being over-represented in gastric corpus cancer when compared to gastritis in the corpus (Tables 2 and 3). A significant decrease in Actinomycetales was observed at the order level in gastric antrum cancer $(15.59 \pm$ $1.83 \%$ vs $0.07 \pm 0.01 \%, p<0.01$ ) (Fig. 4), but not in corpus cancer.

Overall, we found that there are no gross differences in mucosa-associated microbiota profiles between chronic gastritis and H. pylori negative gastric cancer at the phylum level, while major changes occur at relatively lower taxonomic levels. 
Fig. 3 Differential bacteria among three different anatomical sites by LEfSe analysis (LDA scores $>3$ and $p<0.05$ )

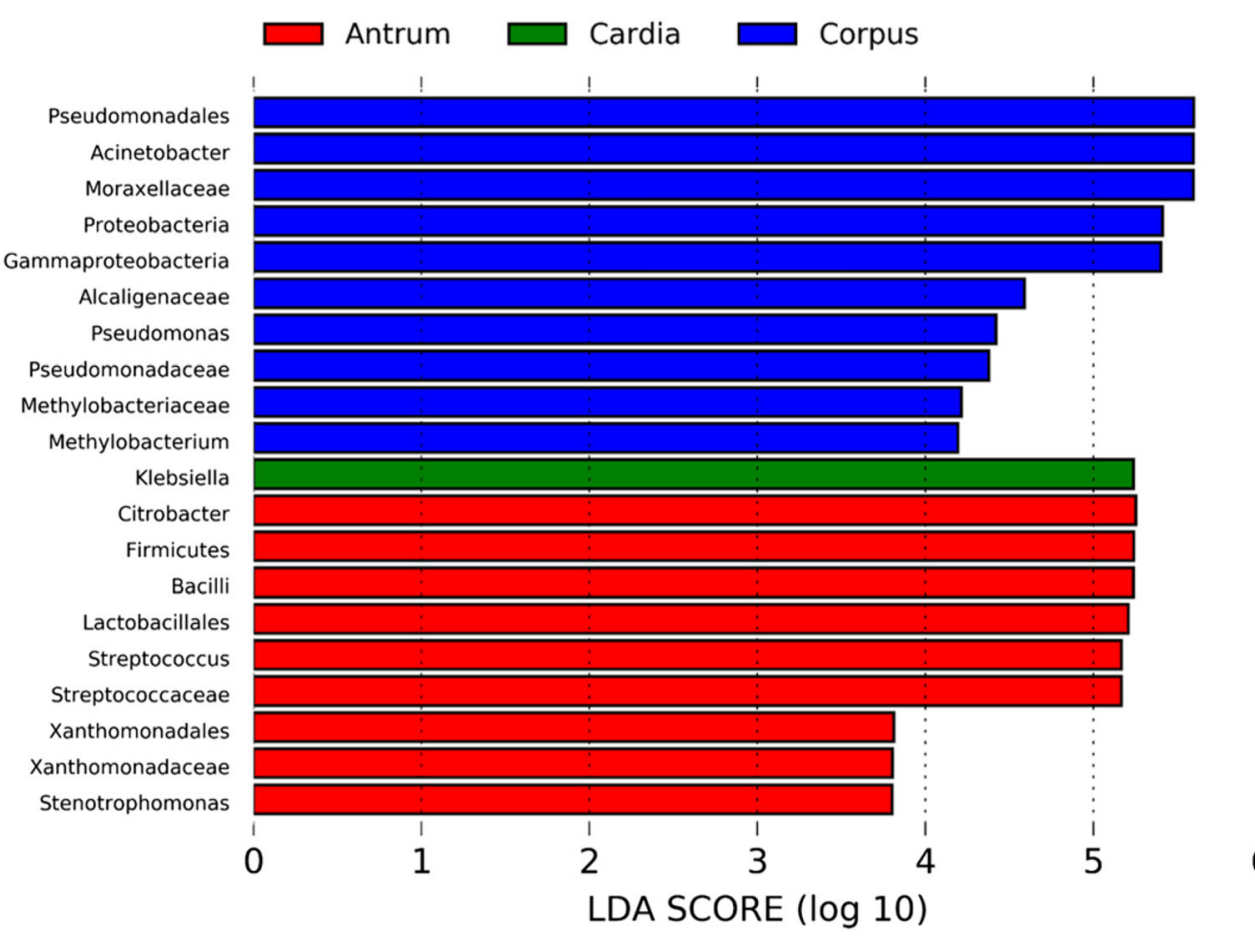

\subsection{Influence of $H$. pylori infection on microbiota profiles in gastric carcinoma}

We next investigated variations in microbial community profiles to assess the potential impact of $H$. pylori infection on the presence of other bacterial flora in gastric cancer. The bacterial communities at the phylum level showed that the abundance of Proteobacteria was slightly higher under H. pylori infection status in both the antrum and corpus (Tables 2 and 3). Nonetheless, dramatic changes also occurred at the order level. Specifically, Campylobacterales (antrum: $45.00 \pm 2.48 \%$; corpus: $84.82 \pm 3.88 \%$ ), instead of Pseudomonadales (antrum: $2.65 \pm 2.97 \%$; corpus: $0.21 \pm 0.26 \%$ ), became the most abundant order in gastric cancer when $H$. pylori was positive (Figs. 4 and 5). These shifts were more noticeable in the corpus, in which all subjects showed a relative abundance of Campylobacterales higher than $90 \%$ (Fig. 5). The over- representation of Actinobacteria in gastric corpus cancer was decreased when infected with $H$. pylori (Table 3 ), but no additional significant changes were observed at the order level.

These results suggest that the influence of $H$. pylori on shaping the gastric cancer-associated microbiota profiles also occurs at lower taxonomic levels.

\subsection{Microbial community structures change from chronic gastritis to gastric carcinoma}

To evaluate alterations in the microbiota community structure from chronic gastritis to gastric carcinoma, we measured bacterial taxonomic richness, alpha diversity and beta diversity. We found that the Chao 1 estimated richness of microbiota in chronic gastritis was not significantly different from that in H. pylroi negative gastric cancer, irrespective of the anatomical sites (Fig. 6a, c). The alpha diversity Shannon index was
Table 2 Relative abundances of major bacterial phyla in gastric antrum cancer

\begin{tabular}{lllll}
\hline Taxonomy & Antrum gastritis (\%) & GC H. pylori (-) (\%) & GC H. pylori (+) (\%) & $P$ value \\
\hline Proteobacteria & 38.03 & 60.22 & 64.71 & 0.35 \\
Firmicutes & 36.94 & 33.11 & 23.04 & 0.76 \\
Actinobacteria & 14.63 & $0.22^{\mathrm{a}}$ & 0.71 & $\mathbf{0 . 0 1}$ \\
Bacteroidetes & 2.95 & $0.93^{\mathrm{a}}$ & 0.98 & 0.46 \\
Fusobacteria & 1.7 & 1.57 & 10.21 & 0.21 \\
\hline
\end{tabular}

The $P$ values are calculated using Mann-Whitney $\mathrm{U}$ test

a Significant difference between $H$. pylori negative gastric antrum cancer patients and antrum-predominant gastritis

$P$ value marked in bold if $<0.05$ 
Table 3 Relative abundances of major bacterial phyla in gastric corpus cancer

\begin{tabular}{lllll}
\hline Taxonomy & Corpus gastritis (\%) & GC H.pylori (-) (\%) & GC H.pylori (+) (\%) & $P$ value \\
\hline Proteobacteria & 80.18 & 82.8 & 91.22 & 0.85 \\
Firmicutes & 7.26 & 0.04 & 1.16 & 0.55 \\
Actinobacteria & 1.69 & $16.70^{\mathrm{a}}$ & $7.47^{\mathrm{b}}$ & 0.51 \\
Bacteroidetes & 1.36 & 0.01 & 0.11 & 0.52 \\
Fusobacteria & 0.38 & 0.01 & 0 & 0.15 \\
\hline
\end{tabular}

The $P$ values are calculated using Mann-Whitney U test

a Significant difference between $H$. pylori negative gastric corpus cancer patients and corpus-predominant gastritis

${ }^{\mathrm{b}}$ Significant difference between H. pylori negative and positive gastric corpus cancer patients markedly decreased in $H$. pylori negative gastric antrum cancer patients in comparison to antrum-predominant gastritis patients (Fig. 6a, $p<0.05$ ), whereas only a downward tendency was observed in corpus mucosa (Fig. 6c). Next, the effect of $H$. pylori infection on microbial richness and alpha diversity was determined in gastric cancer. Microbial richness tended to increase under $H$. pylori infection status, but was statistically significant only in gastric corpus cancer (Fig. $6 \mathrm{a}, \mathrm{c})$. Alpha diversity tended to increase when infected with H. pylori both in gastric antrum and corpus cancer (Fig. 6a, c), but it did not reach statistical significance $(p>0.05)$.

Beta diversity analysis revealed an obvious separation between chronic gastritis and gastric cancer in both the antrum and corpus, but further distinguishing according to $H$. pylori infection status depended on the anatomical tumor sites (Fig. $6 \mathrm{c}, \mathrm{d})$. Gastric corpus cancer patients clustered separately based on the abundance of H. pylori (Fig. 6d), but not in patients with gastric antrum cancer (Fig. 6c).

These results suggest that there is no obvious alteration in microbial alpha diversity and richness in different stomach phenotypes, including chronic gastritis and $H$. pylori negative or positive gastric cancer. The endogenous bacterial community is disturbed during the initiation and development of gastric cancer, whilst the role of $H$. pylori in the progression of malignant tumors is limited, or at least needs to be further determined.

\subsection{Specific microbial taxa associated with gastric cancer}

To identify the most relevant taxa responsible for each clinical diagnosis, we next performed LEfSe analysis at the order level and above, which has been validated for high-dimensional biomarker discovery [19]. For patients with antrum predominant gastritis and gastric antrum cancer, this analysis identified 12 taxa, including 5 orders, which were differentially abundant among the three groups. In $H$. pylori negative gastric cancer patients, an enrichment in Proteobacteria and Firmicutes taxa was observed, including the class Gammaproteobacteria with its specific order Peudomonodales, and the class Erysipelotrichia with its specific order Erysipelotrichales. However, in H. pylori positive gastric cancer patients, Neisseriales was the only identified enriched order within the class Betaproteobacteria. Additionally, Actinomycetales, Enterobacteriales and Pasteurellales were more abundant in the microbiota of patients with antrum predominant gastritis (Fig. 7a).

For patients with corpus predominant gastritis and gastric corpus cancer, there were also 12 specific taxa that were differentially abundant among the three different clinical diagnoses. In $H$. pylori negative gastric cancer patients, we only found that Gammaproteobacteria with its specific order Peudomonodales were significantly abundant. When it comes to $H$. pylori positive gastric cancer subjects, except Proteobacteria taxa with its specific order Campylobacterales, an enrichment in the phylum Bacteroidetes with its specific order Bacteroidales was observed (Fig. 7b). Moreover, Enterobacteriales, Actinomycetales and Burkholderiales were more abundant in patients with corpus predominant gastritis.

These analyses revealed the most relevant taxa responsible for differences between clinical diagnoses, and that the abundance of specific taxa depends on $H$. pylori infection status.

\section{Discussion}

Through increasing technological developments, the microbial profiles in tumor environments are now being unraveled [20-23]. Here, we explored distinct gastric mucosaassociated microbiota profiles among different anatomical sites during gastritis, the shift of the microbiome community during gastric cancer progression, and the influence of H. pylori infection on the microbial community in gastric cancer. Our findings add to the understanding of associations between dysbiosis and gastric cancer development.

An intricate interaction between acid secretion and bacterial growth is a crucial determinant of $H$. pylori infection and gastritis progression [24-26]. The differential levels of acid secretion contribute to the distinct niches of the gastric antrum and corpus for microbial colonization [26]. In subjects with high acid production, $H$. pylori has been found to particularly 
Fig. 4 Microbial compositional analysis of patients with antrum predominant gastritis and gastric antrum cancer. a Heatmap of gastric mucosa-associated microbiota with relative abundance over $1 \%$ in all subjects. b Bar plots of relative abundance of gastric mucosa-associated microbiota. $\mathbf{c}$ Representative microbiota with significant differences $(p<0.05), *$ Significant difference between antrumpredominant gastritis and H. pylori negative gastric antrum cancer patients. " Significant difference between $H$. pylori negative and positive gastric antrum cancer patients a
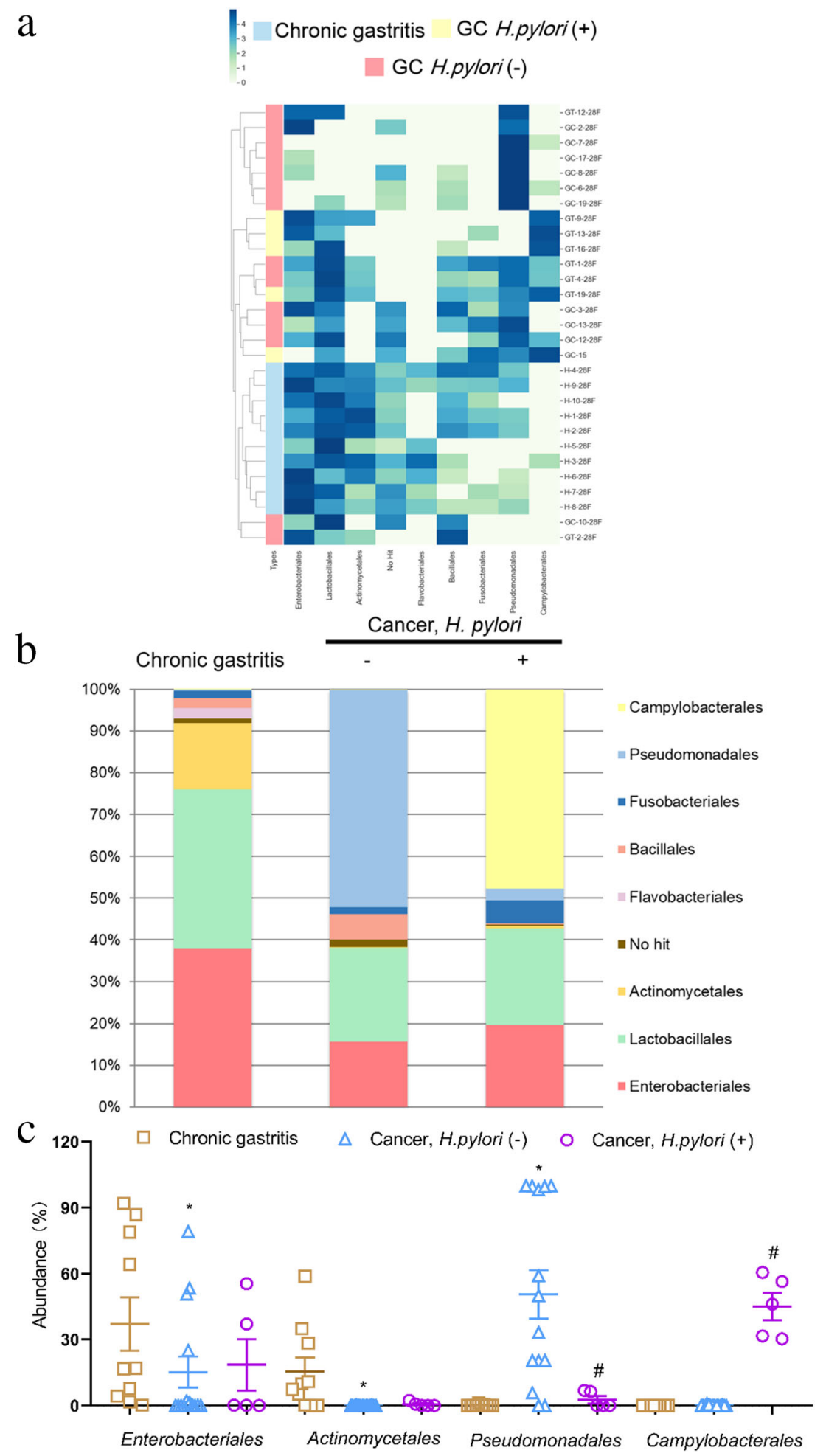

colonize the gastric antrum and to lead to antrum-predominant gastritis. However, when acid secretion is impaired, the location of gastritis is dominant in the corpus [27]. Based on these observations, a detailed assessment of the microbial community at different anatomical locations is considered important in order to understand the role of the microbiota community in the stomach. Our results revealed similar gastric microbiota communities during chronic gastritis in three different anatomical sites. These findings are consistent with previously reported results indicating that the microbial diversity and richness of these three anatomical positions were similar [23] and that no significant correlation was observed between 
a

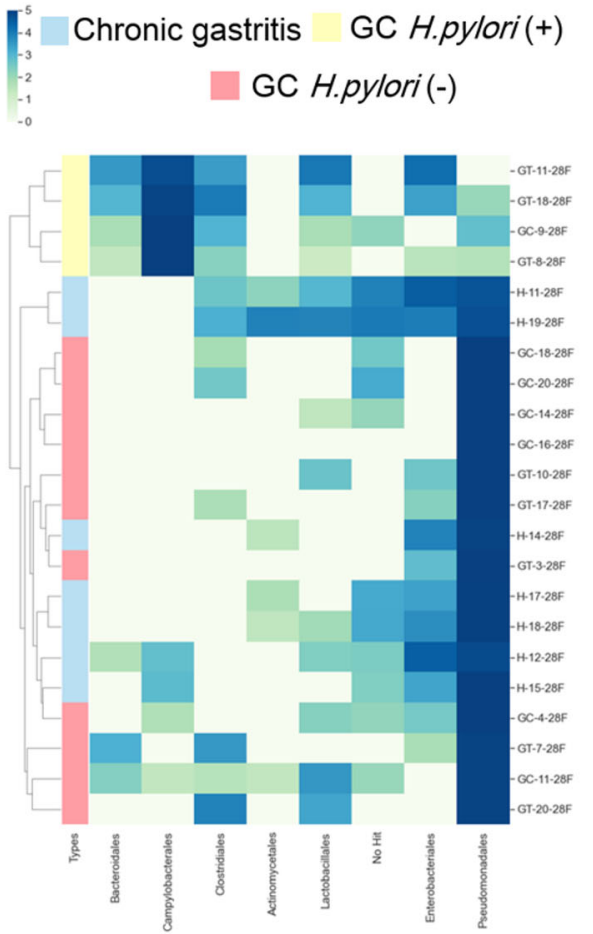

b
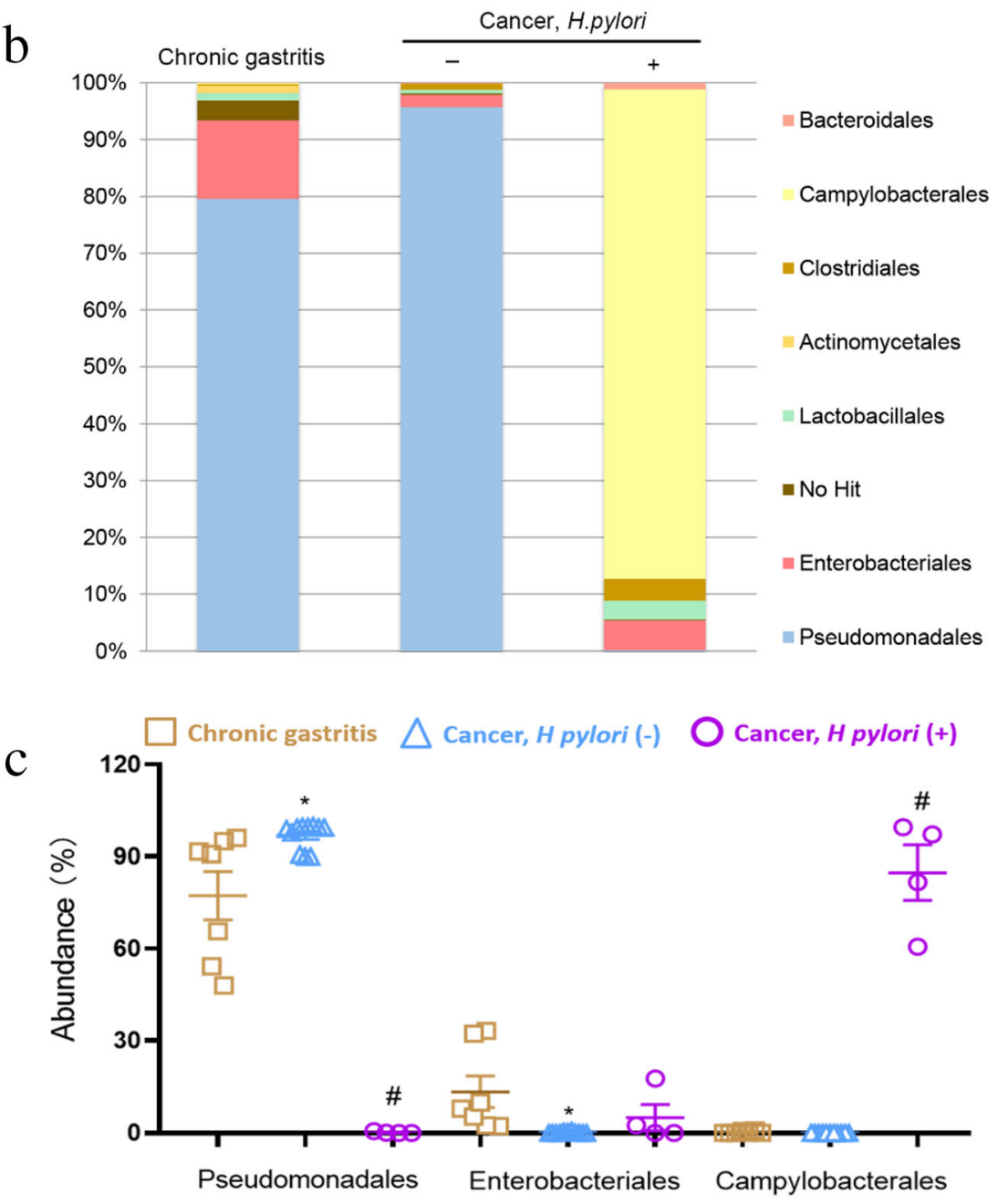
Fig. 5 Microbial composition analysis of patients with corpus predominant gastritis and gastric corpus cancer. a Heatmap of gastric mucosa-associated microbiota with relative abundance over $1 \%$ in all subjects. b Bar plots of relative abundances of gastric mucosaassociated microbiota. $\mathbf{c}$ Representative microbiota with significant differences $(p<0.05)$, * Significant difference between corpus-predominant gastritis and $H$. pylori negative gastric corpus cancer patients. \# Significant difference between H. pylori negative and positive gastric corpus cancer patients phylotype distribution and anatomical location in Chinese symptomatic upper gastrointestinal disease patients [28].

Changes in gastric acid secretion, local immune responses and nutrient availability contribute to the disruption of homeostasis, leading to disturbances in microbial composition, structure and function, resulting in the colonization and overgrowth of none-H. pylori bacteria [29]. The complexity of microbiota can be discerned based on biological similarity in order to analyze phylogenetic and evolutionary relationships between different phenotypes. Analysis at the phylum level may provide a general overview of distinguished characteristics of
Fig. 6 Microbial community structure analysis from chronic gastritis to gastric cancer. a Microbial alpha diversity and richness analyses between antrum predominant gastritis and gastric antrum cancer. b PCA analysis between antrum predominant gastritis and gastric antrum cancer. c Microbial alpha diversity and richness analyses between corpus predominant gastritis and gastric corpus cancer d PCA analysis between corpus predominant gastritis and gastric corpus cancer a
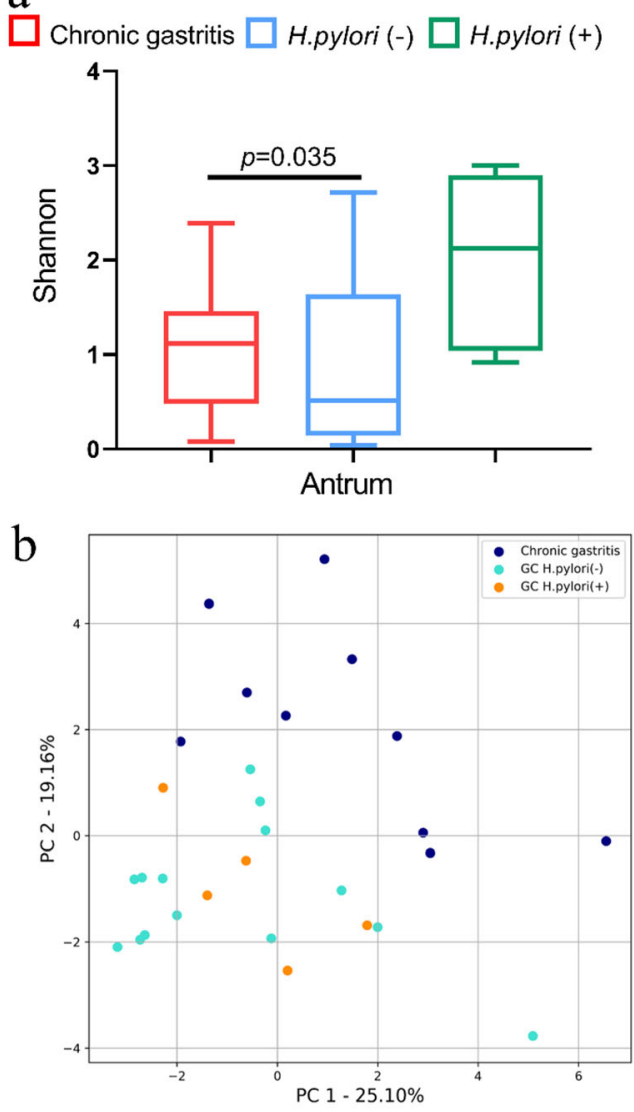

$\mathrm{C}$

$\square$ Chronic gastritis $\square$ H.pylori (-) $\square$ H.pylori (+)

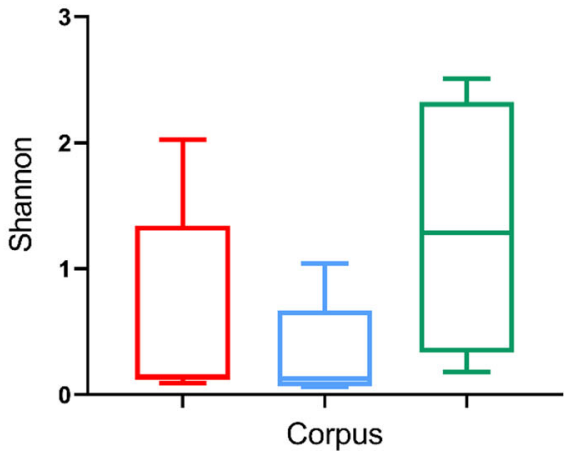

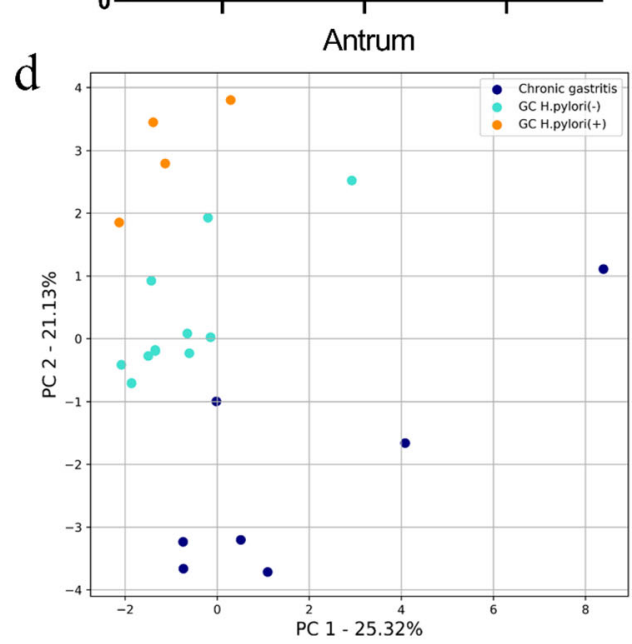

Chronic gastritis $\square$ H.pylori (-) $\square$ H.pylori (+)

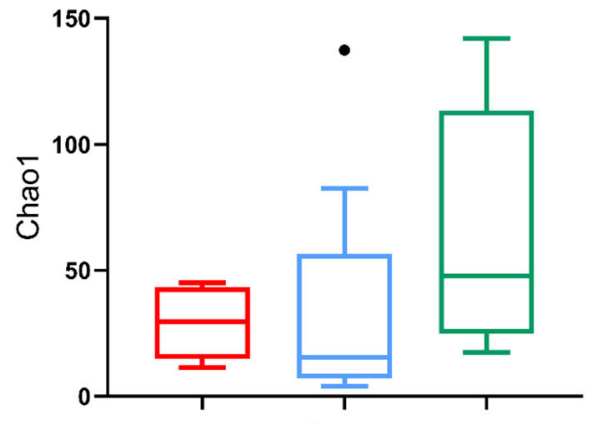

d

Chronic gastritis $\square$ H.pylori (-) $\square$ H.pylori (+)

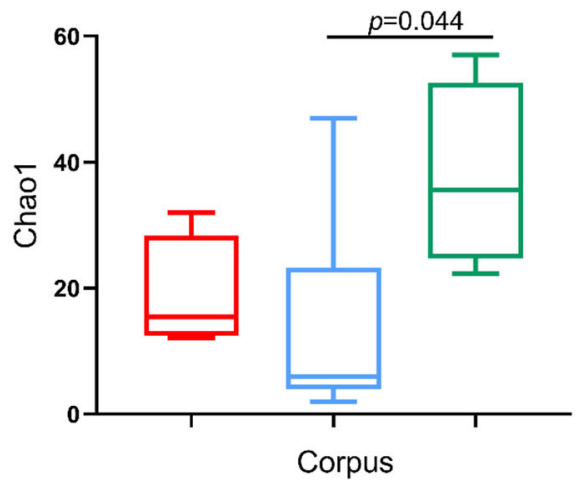


Fig. 7 Influence of $H$. pylori infection on the specific taxa associated with different clinical diagnoses based on LEfSe analysis (LDA scores $>3$ and $p<0.05)$. a Specific microbial taxa associated with antrum predominant gastritis and gastric antrum cancer. b Specific microbial taxa associated with corpus predominant gastritis and gastric corpus cancer a
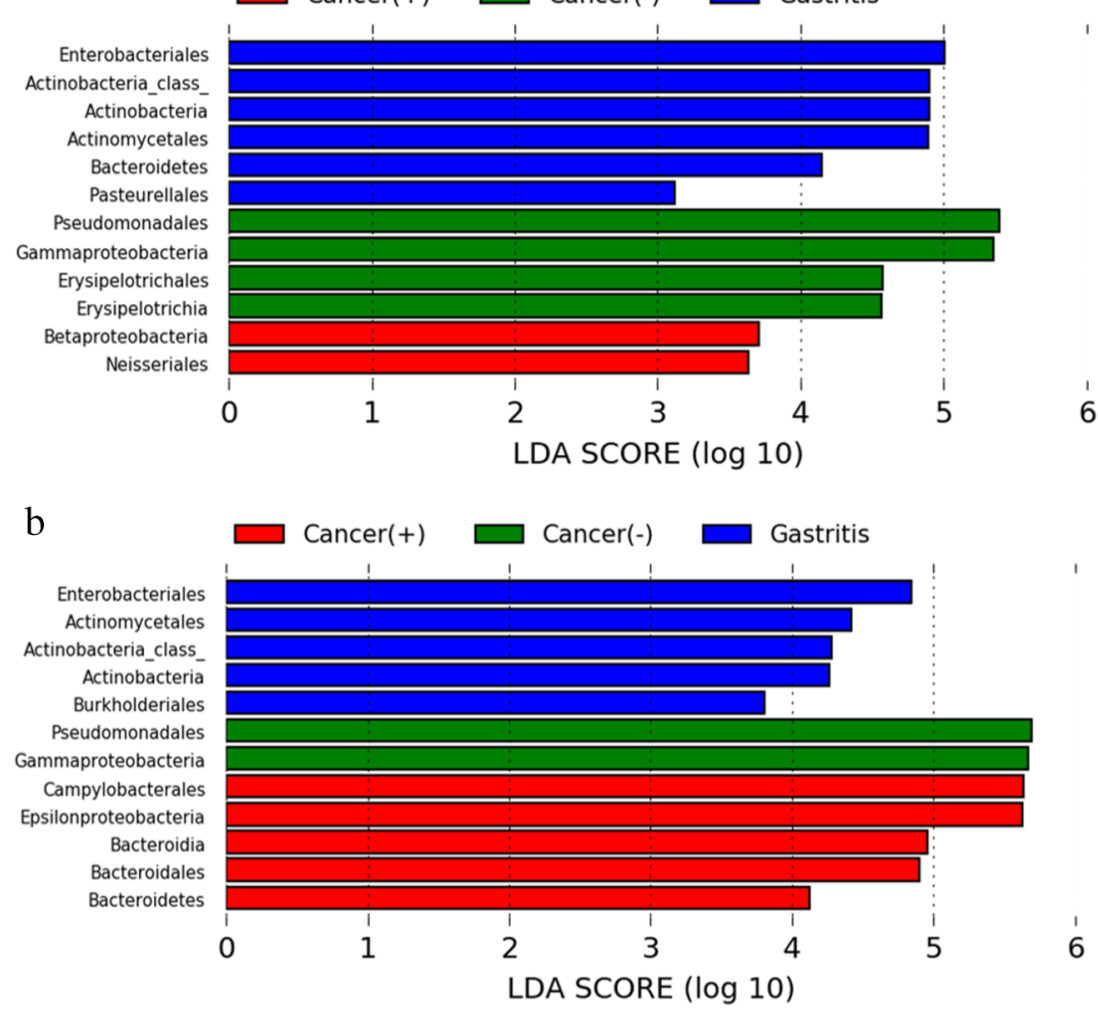

different diseases at a relatively higher taxonomic level. Our present study showed that the influence of $H$. pylori infection on gastric cancer mucosa-associated microbial composition at the phylum level was limited, and that microbiota in cancerous and non-cancerous tissues were both dominated by Proteobacteria, Firmicutes, Actinobacteria, Bacteroidetes and Fusobacteria, as reported before [21-23]. Significant differences among different groups mainly occurred at a relatively lower taxonomic level. Chronic gastritis was mainly characterized by enrichment of lactic acid producing bacteria such as Lactobacillus and commensals of the oral cavity and lower gastrointestinal tract including Streptococcus, Citrobacter and Klebsiella [30, 31]. These findings differ from previous studies, which reported that Citrobacter and Klebsiella were more abundant in gastric cancer and were associated with gastric carcinogenesis $[22,32,33]$. This discrepancy may be due to differences in ethnicity, sample type, dietary habits and/or environmental factors. When progressed into $H$. pylori negative gastric cancer, the microbial profiles shifted dramatically with an absolute abundance of Pseudomonadales, in which Acinetobacter $s p$ was the only prevalent species. Consistent with our data, a previous report using public cancer genome sequencing data showed that Pseudomonas was the stomach adenocarcinoma-associated bacterium when excluding contamination attribution [34]. In contrast, when infected with H. pylori, Campylobacterales was dominating the mucosaassociated community in gastric cancer. At the species level,
H. pylori was highly abundant within this order, especially in mucosa of the corpus. It is noteworthy that the nitrosating or nitrate-reducing bacterium Neisseriales was more abundant in gastric antrum cancer, which can promote the production of $\mathrm{N}$-nitroso compounds (NOC) which, in turn, have been considered as potent carcinogens [32, 35, 36]. Enrichment of Betaproteobacteria and Gammaproteobacteria in gastric cancer indicated that none-H. pylori Proteobacteria may be closely associated with gastric carcinogenesis.

In our study, no significant alterations in local microbial community richness (evaluated by Chao 1 ) and alpha diversity (evaluated by Shannon index, except in the antrum) were observed during the development of gastritis to $H$. pylori negative gastric cancer. In addition, we found that the presence of H. pylori did not affect the species richness (except in the corpus) and alpha diversity of the gastric cancer community. This observation is not completely consistent with previous reports, showing significant reductions in microbial diversity and species richness in gastric cancer compared with chronic gastritis [22, 23]. Another cohort, in which H. pylori infection status was not considered, revealed that gastric cancer exhibited a significantly increased microbial diversity and species richness compared to non-cancerous tissues [21]. Factors that may provide an explanation to these discrepancies include variations in $\mathrm{H}$. pylori status and virulence, sample type, dietary factors, as well as sequencing techniques and analysis methods used. Additionally, due to the different stages of 
gastric cancer development in our analysis cohort, a high degree of inter-subject variability may exist. Statistically significant differences among groups may, therefore, become apparent when the sample sizes are expanded. Nevertheless, beta diversity analysis revealed distinct microbiota structures between chronic gastritis and gastric cancer groups. The nichespecific microbial communities observed may reflect that multiple bacteria may have gradually adapted to diseasespecific microenvironments. The microbial community structure could be diversified in gastric corpus cancer according to H. pylori infection status, but not in the antrum. This may be due to the different ambient $\mathrm{pH}$ levels between the corpus and antrum. As indicated above, the $\mathrm{pH}$ of the corpus may be more vulnerable to $H$. pylori infection than the $\mathrm{pH}$ of the antrum.

It should be noted that H.pylpri positive gastric corpus cancer showed a significant increase in species richness compared to the negative group. The alpha diversity also exhibited an increased tendency from $H$. pylori negative to positive gastric corpus cancer, although without statistical significance. This finding is supported by previous reports using paired cancerous and non-cancerous tissues, indicating that the H. pylori sequencing-positive group showed a significant increase in alpha diversity in comparison to the negative group [21]. Another study substantiated the view that $H$. pylori can markedly influence the diversity of gastric cancer [37]. Also, a mouse study indicated an increased bacterial diversity in $H$. pylori infected animals, which appears to correlate with observations made in infected humans [38]. A possible explanation for this phenomenon is that chronic infection of $H$. pylori induces an inflammatory cascade and gastric atrophy, a condition related to an impaired secretion of gastric acid leading to an increase in $\mathrm{pH}$, consequently decreasing the load of $H$. pylori and its expression pattern, all together rendering a more tolerable environment for the colonization and outgrowth of nonH. pylori microbiota [39]. The suicide journey hypothesis of $H$. pylori through gastric carcinogenesis is supported by many reports, showing that the abundance of H. pylori is lower in any pre-malignant lesion or gastric cancer compared to that in non-malignant gastric mucosa $[20,22,40]$. Thus, H. pylori may be the predominant microorganism during the initial stages of gastric carcinogenesis, after which it becomes a minor component and substituted by new dominant microbiota, which favor gastric cancer development at later stages [41, 42]. What we observed in our study may lie in the earlier period of this shift. Understanding the timing of this evolution of gastric microbiota may provide insight into the intricate role of microbiota in tumor development. The best time at which to eradicate $H$. pylori and to administer a proton pump inhibitor should be determined in order to halt gastric carcinogenesis.
Our future studies will be aimed at analyzing $H$. pylori positive gastritis subjects and focusing on microbial changes from gastritis to gastric cancer with the presence of $H$. pylori, and fully clarify the role of $H$. pylori in gastric carcinogenesis. The current study was based on a relatively small number of patients, especially $H$. pylori positive gastric cancer patients. Future prospective follow-up studies using large populations are required to determine the landscape of gastric microbiota along with the development of gastric cancer. Nevertheless, our study provides a foundation for a more comprehensive understanding of gastric cancer mucosa-associated microbiota and the complex interplay between $H$. pylori and other microbiota, which may shed light on the pathogenesis, diagnosis, treatment and prevention of gastric cancer.

Supplementary Information The online version contains supplementary material available at https://doi.org/10.1007/s13402-021-00596-y.

Acknowledgements This work was supported by the National Natural Science Foundation of China (81772620) and Tianjin Science and Technology Major Project of Chronic Diseases Prevention and Control (17ZXMFSY00130).

Author contributions Conceptualization, formal analysis, investigation, writing-original draft: Yilin Deng. Resources: Xuewei Ding, Gang Zhao, Bowen Ding, Xianhao Wang. Software: Qingyuan Song. Formal analysis: Lei Han. Conceptualization, funding acquisition, project administration, writing-review and editing: Xishan Hao, Hui Li.

Data and code availability The data and code used to support the findings of this study are available from the corresponding author upon request.

\section{Declarations}

Ethical approval All procedures followed were in accordance with the ethical standards of the responsible committee on human experimentation (institutional and national) and with the Helsinki Declaration of 1964 and its later amendments or comparable ethical versions.

Informed consent Written informed consent was obtained from all individuals included in this study.

Conflict of interest Authors declare no potential competing financial interest.

Open Access This article is licensed under a Creative Commons Attribution 4.0 International License, which permits use, sharing, adaptation, distribution and reproduction in any medium or format, as long as you give appropriate credit to the original author(s) and the source, provide a link to the Creative Commons licence, and indicate if changes were made. The images or other third party material in this article are included in the article's Creative Commons licence, unless indicated otherwise in a credit line to the material. If material is not included in the article's Creative Commons licence and your intended use is not permitted by statutory regulation or exceeds the permitted use, you will need to obtain permission directly from the copyright holder. To view a copy of this licence, visit http://creativecommons.org/licenses/by/4.0/. 


\section{References}

1. J. Lloyd-Price, G. Abu-Ali, C. Huttenhower, The healthy human microbiome. Genome Med. 8, 51 (2016)

2. N.H.W. Group, J. Peterson, S. Garges, M. Giovanni, P. McInnes, L. Wang, J.A. Schloss, V. Bonazzi, J.E. McEwen, K.A. Wetterstrand, C. Deal, C.C. Baker, V. Di Francesco, T.K. Howcroft, R.W. Karp, R.D. Lunsford, C.R. Wellington, T. Belachew, M. Wright, C. Giblin, H. David, M. Mills, R. Salomon, C. Mullins, B. Akolkar, L. Begg, C. Davis, L. Grandison, M. Humble, J. Khalsa, A.R. Little, H. Peavy, C. Pontzer, M. Portnoy, M.H. Sayre, P. StarkeReed, S. Zakhari, J. Read, B. Watson, M. Guyer, The NIH Human Microbiome Project. Genome Res. 19, 2317-2323 (2009)

3. Fredrik Ba"ckhed, Ruth E. Ley, Justin L. Sonnenburg, Daniel A. Peterson, J.I. Gordon. Host-bacterial mutualism in the human intestine. Science 307, 1915-1920 (2005)

4. I. Cho, M.J. Blaser, The human microbiome: At the interface of health and disease. Nat. Rev. Genet. 13, 260-270 (2012)

5. J.Robin Warren, Barry Marshall, B.A.Scoggins, J.A.Whitworth, J.P.Coghlan, D.A.Denton, R.T.Mason. Unidentified curved bacilli on gastirc epithelium in active chronic gastritis. The Lancet $\mathbf{1}$, 1273-1275 (1983)

6. A. Sheh, J.G. Fox, The role of the gastrointestinal microbiome in helicobacter pylori pathogenesis. Gut Microbes 4, 505-531 (2013)

7. W.S. Garrett, Cancer and the microbiota. Science 348, 80-86 (2015)

8. J. Ni, G.D. Wu, L. Albenberg, V.T. Tomov, Gut microbiota and IBD: Causation or correlation? Nat. Rev. Gastroenterol. Hepatol. 14, 573-584 (2017)

9. A. Tripathi, J. Debelius, D.A. Brenner, M. Karin, R. Loomba, B. Schnabl, R. Knight, The gut-liver axis and the intersection with the microbiome. Nat. Rev. Gastroenterol. Hepatol. 15, 397-411 (2018)

10. T. Van de Wiele, J.T. Van Praet, M. Marzorati, M.B. Drennan, D. Elewaut, How the microbiota shapes rheumatic diseases. Nat. Rev. Rheumatol. 12, 398-411 (2016)

11. T.C. Fung, C.A. Olson, E.Y. Hsiao, Interactions between the microbiota, immune and nervous systems in health and disease. Nat. Neurosci. 20, 145-155 (2017)

12. F. Bray, J.S. Ren, E. Masuyer, J. Ferlay, Global estimates of cancer prevalence for 27 sites in the adult population in 2008. Int. J. Cancer 132, 1133-1145 (2013)

13. M.T. Abreu, R.M. Peek Jr., Gastrointestinal malignancy and the microbiome. Gastroenterol. 146, 1534-1546 e1533 (2014)

14. P. Karimi, F. Islami, S. Anandasabapathy, N.D. Freedman, F. Kamangar, Gastric cancer: Descriptive epidemiology, risk factors, screening, and prevention. Cancer Epidemiol. Biomark. Prev. 23, 700-713 (2014)

15. J.L. Lofgren, M.T. Whary, Z. Ge, S. Muthupalani, N.S. Taylor, M. Mobley, A. Potter, A. Varro, D. Eibach, S. Suerbaum, T.C. Wang, J.G. Fox, Lack of commensal flora in helicobacter pylori-infected INS-GAS mice reduces gastritis and delays intraepithelial neoplasia. Gastroenterol. 140, 210-220 (2011)

16. K. Lertpiriyapong, M.T. Whary, S. Muthupalani, J.L. Lofgren, E.R. Gamazon, Y. Feng, Z. Ge, T.C. Wang, J.G. Fox, Gastric colonisation with a restricted commensal microbiota replicates the promotion of neoplastic lesions by diverse intestinal microbiota in the helicobacter pylori INS-GAS mouse model of gastric carcinogenesis. Gut 63, 54-63 (2014)

17. P. Correa, A human model of gastric carcinogenesis. Cancer Res. 48, 3554-3560 (1988)
18. J. Kim, N. Kim, H.J. Jo, J.H. Park, R.H. Nam, Y.J. Seok, Y.R. Kim, J.S. Kim, J.M. Kim, J.M. Kim, D.H. Lee, H.C. Jung, An appropriate cutoff value for determining the colonization of helicobacter pylori by the pyrosequencing method: Comparison with conventional methods. Helicobacter 20, 370-380 (2015)

19. N. Segata, J. Izard, L. Waldron, D. Gevers, L. Miropolsky, W.S. Garrett, C. Huttenhower, Metagenomic biomarker discovery and explanation. Genome Biol. 12, R60 (2011)

20. J. Dicksved, M. Lindberg, M. Rosenquist, H. Enroth, J.K. Jansson, L. Engstrand, Molecular characterization of the stomach microbiota in patients with gastric cancer and in controls. J. Med. Microbiol. 58, 509-516 (2009)

21. X.H. Chen, A. Wang, A.N. Chu, Y.H. Gong, Y. Yuan, Mucosaassociated microbiota in gastric cancer tissues compared with noncancer tissues. Front. Microbiol. 10, 1261 (2019)

22. R.M. Ferreira, J. Pereira-Marques, I. Pinto-Ribeiro, J.L. Costa, F. Carneiro, J.C. Machado, C. Figueiredo, Gastric microbial community profiling reveals a dysbiotic cancer-associated microbiota. Gut 67, 226-236 (2018)

23. O.O. Coker, Z. Dai, Y. Nie, G. Zhao, L. Cao, G. Nakatsu, W.K. Wu, S.H. Wong, Z. Chen, J.J.Y. Sung, J. Yu, Mucosal microbiome dysbiosis in gastric carcinogenesis. Gut 67, 1024-1032 (2018)

24. K.M. Brawner, C.D. Morrow, P.D. Smith, Gastric microbiome and gastric cancer. Cancer J. 20, 211-216 (2014)

25. J. Li, G.I. Perez Perez, Is there a role for the non-helicobacter pylori bacteria in the risk of developing gastric Cancer? Int. J. Mol. Sci. 19, 1353-1362 (2018)

26. M. Amieva, R.M. Peek Jr., Pathobiology of helicobacter pyloriinduced gastric cancer. Gastroenterol. 150, 64-78 (2016)

27. J.G. Kusters, A.H. van Vliet, E.J. Kuipers, Pathogenesis of helicobacter pylori infection. Clin. Microbiol. Rev. 19, 449-490 (2006)

28. Elisabeth M. Bik, Paul B. Eckburg, Steven R. Gill, Karen E. Nelson, Elizabeth A. Purdom, Fritz Francois, Guillermo PerezPerez, Martin J. Blaser, D.A. Relman. Molecular analysis of the bacterial microbiota in the human stomach. Proc. Natl. Acad. Sci. U. S. A. 103, 732-737 (2006)

29. J.L. Espinoza, A. Matsumoto, H. Tanaka, I. Matsumura, Gastric microbiota: An emerging player in helicobacter pylori-induced gastric malignancies. Cancer Lett. 414, 147-152 (2018)

30. Jinzhe Liu, Yan Xue, Liya Zhou. Detection of gastritis-associated pathogens by culturing of gastric juice and mucosa. Int. J. Clin. Exp. Pathol. 11, 2214-2220 (2018)

31. V. Passet, S. Brisse, Description of Klebsiella grimontii sp. nov. Int. J. Syst. Evol. Microbiol. 68, 377-381 (2018)

32. S. J. Forsythe, J. M. Dolby, A. D. B. Websters, J.A. Cole. Nitrateand nitrite-reducing bacteria in the achlorhydric stomach. J . Med. Microbiol. 25, 253-259 (1988)

33. E. Dias-Jacome, D. Libanio, M. Borges-Canha, A. Galaghar, P. Pimentel-Nunes, Gastric microbiota and carcinogenesis: The role of non-helicobacter pylori bacteria - a systematic review. Rev. Esp. Enferm. Dig. 108, 530-540 (2016)

34. K.M. Robinson, J. Crabtree, J.S. Mattick, K.E. Anderson, J.C. Dunning Hotopp, Distinguishing potential bacteria-tumor associations from contamination in a secondary data analysis of public cancer genome sequence data. Microbiome 5, 9 (2017)

35. M.A. Abateni Ayanaba, Microbial formation of nitrosamines in vitro. Appl. Microbiol. 25, 862-868 (1973)

36. S.H. Sohn, N. Kim, H.J. Jo, J. Kim, J.H. Park, R.H. Nam, Y.J. Seok, Y.R. Kim, D.H. Lee, Analysis of gastric body microbiota by pyrosequencing: Possible role of bacteria other than helicobacter pylori in the gastric carcinogenesis. J. Cancer Prev. 22, 115-125 (2017) 
37. L. Wang, J. Zhou, Y. Xin, C. Geng, Z. Tian, X. Yu, Q. Dong, Bacterial overgrowth and diversification of microbiota in gastric cancer. Eur. J. Gastroenterol. Hepatol. 28, 261-266 (2016)

38. J.P. Fischer, H. Cypionka, Vaccination prevents helicobacter pylori-induced alterations of the gastric flora in mice. FEMS Microbiol. Ecol. 55, 186-194 (2006)

39. J.M. Noto, R.M. Peek Jr., The gastric microbiome, its interaction with helicobacter pylori, and its potential role in the progression to stomach cancer. PLoS Pathog. 13, e1006573 (2017)

40. Y.Y. Hsieh, S.Y. Tung, H.Y. Pan, C.W. Yen, H.W. Xu, Y.J. Lin, Y.F. Deng, W.T. Hsu, C.S. Wu, C. Li, Increased abundance of Clostridium and Fusobacterium in gastric microbiota of patients with gastric cancer in Taiwan. Sci. Rep. 8, 158 (2018)
41. P.P. de Assumpcao, T.M.T. Araujo, P.B. de Assumpcao, W.F. Barra, A.S. Khayat, C.B. Assumpcao, G. Ishak, D.N. Nunes, E. Dias-Neto, L.G.V. Coelho, Suicide journey of H. pylori through gastric carcinogenesis: the role of non- $H$. pylori microbiome and potential consequences for clinical practice. Eur. J. Clin. Microbiol. Infect. Dis. 38, 1591-1597 (2019)

42. G.L. Hold, R. Hansen, Impact of the gastrointestinal microbiome in health and disease: Co-evolution with the host immune system. Curr. Top. Microbiol. Immunol. 421, 303-318 (2019)

Publisher's note Springer Nature remains neutral with regard to jurisdictional claims in published maps and institutional affiliations. 